\title{
THE CURVATURE OF LEVEL CURVES
}

\author{
BY \\ DOROTHY BROWNE SHAFFER( $\left.{ }^{1}\right)$
}

\begin{abstract}
Sharp bounds are derived for the curvature of level curves of analytic functions in the complex plane whose logarithmic derivative has the representation $c /(w-g(w))$, where $g(w)$ is analytic for $|w|>a$ and $|g(w)| \leqq a, c$ real. These results are applied in particular to lemniscates and sharpened for the level curves of lacunary polynomials. Extensions to the level curves of Green's function and rational functions are indicated.
\end{abstract}

1. Introduction. In this paper sharp bounds are derived for the curvature of various classes of level curves of analytic functions in the complex plane. In $\$ \S 2$ and 3 a considerably simplified proof is given for the estimates of the curvature of lemniscates, the level curves of Green's function and rational functions derived by the author in previous publications [4], [5]. In $\$ 4$ new estimates for the curvature of the level lines of lacunary polynomials and their orthogonal trajectories are derived. The methods used combine the author's previous calculations with an application of a new coincidence lemma due to Rubinstein and Walsh [3]. These estimates are sharp. Applications to the special lemniscate of radius one are indicated in the final section.

2. Estimates of the curvature of the level curves of special functions. In this section bounds are derived for curvature of level curves $V_{\mu}:\{w|| P(w) \mid=\mu, \mu>0\}$ of analytic functions $P(w)$ whose logarithmic derivative has a special representation. The following section deals with the determination of the classes of functions which satisfy the required representation.

THEOREM 1. Let $P(w)$ be a function such that for $|w|>$ a the function $P^{\prime}(w) / P(w)$ has the representation

(1) $P^{\prime}(w) / P(w)=c /(w-g(w))$, c real, $g(w)$ analytic for $|w|>a,|g(w)| \leqq a$;

then the curvature $K(w)$ at $w$ of the level curve $V_{\mu}$ satisfies the following bounds:

$$
\begin{aligned}
& (\operatorname{sgn} c) K(w) \leqq 1 /(h-a), \\
& |w|=h>a, \\
& (\operatorname{sgn} c) K(w) \geqq 1 /(h+a) \\
& \text { for } h \geqq 3 a \text {, } \\
& (\operatorname{sgn} c) K(w) \geqq 2 \frac{\left(2\left(h^{2}-a^{2}\right)\right)^{1 / 2}-h}{h^{2}-a^{2}} \\
& \text { for } h \leqq 3 a \text {. }
\end{aligned}
$$

Presented to the Society, March 27, 1970 under the title The curvature of level curves of lacunary polynomials and Green's function; received by the editors May 18, 1970.

AMS 1969 subject classifications. Primary 3010.

Key words and phrases. Level line, lemniscate, curvature, lacunary polynomial, convexity.

( $\left.{ }^{1}\right)$ The author gratefully acknowledges the support of the National Science Foundation Grant GP-23504 and Science Faculty Fellowship.

Copyright (C) 1971, American Mathematical Society 
Proof. Study's formula for the curvature of the image of a circle under an analytic transformation is used as the starting point for the calculations, i.e.

$$
K=\operatorname{Re}\left\{1+z f^{\prime \prime}(z) / f^{\prime}(z)\right\} /\left|z f^{\prime}(z)\right| .
$$

By applying this to the inverse function $f=P^{-1}$ the following expression for the curvature $K(w)$ of $V_{\mu}$ at the point $w$ is obtained:

$$
K(w)=\operatorname{Re}\left\{1-\frac{P(w) P^{\prime \prime}(w)}{P^{\prime}(w)^{2}}\right\}\left|\frac{P^{\prime}(w)}{P(w)}\right| .
$$

For a function with the given representation (1) this yields

$$
(\operatorname{sgn} c) K(w)=\operatorname{Re}\left\{1-g^{\prime}(w)\right\} / s \quad \text { where } s=|w-g(w)| .
$$

The bounds of the curvature follow in the form

$$
\left(1-\left|g^{\prime}(w)\right|\right) / s \leqq(\operatorname{sgn} c) K(w) \leqq\left(1+\left|g^{\prime}(w)\right|\right) / s .
$$

The next step in the proof is the application of standard inequalities for the absolute value of the derivative of a bounded analytic function [1, p. 168]. Under the hypothesis of our theorem $g(w)$ is analytic for $|w|>a,|g(w)| \leqq a$, and

$$
\left|g^{\prime}(w)\right| \leqq\left(a^{2}-|g(w)|^{2}\right) /\left(|w|^{2}-a^{2}\right) .
$$

It follows that

$$
\frac{|w|^{2}-2 a^{2}+|g|^{2}}{s\left(|w|^{2}-a^{2}\right)} \leqq(\operatorname{sgn} c) K(w) \leqq \frac{|w|^{2}-|g|^{2}}{s\left(|w|^{2}-a^{2}\right)}
$$

In order to express the bounds (7) as functions of a single variable the identity $g=g-w+w$ is used. This yields

$$
|w|^{2}-|g|^{2}=-s^{2}+2 \operatorname{Re}\{(\overline{g-w}) w\} .
$$

For the right-hand inequality we obtain

$$
\begin{aligned}
(\operatorname{sgn} c) K & \leqq \frac{-s+2 h \cos \arg (\overline{w-g}) w}{h^{2}-a^{2}}, \quad|w|=h, \\
& \leqq \frac{-s+2 h}{h^{2}-a^{2}} .
\end{aligned}
$$

The bound (2a) is obtained by substitution of the smallest value of $s$, such that the hypothesis $|g(w)| \leqq a$ is satisfied, i.e. $s=h-a$.

The left-hand inequality is rewritten in the form

$$
(\operatorname{sgn} c) K \geqq \frac{2\left(|w|^{2}-a^{2}\right)+|g|^{2}-|w|^{2}}{s\left(|w|^{2}-a^{2}\right)} .
$$

By application of the identity (8) this is reduced to

$$
(\operatorname{sgn} c) K \geqq \frac{2}{s}+\frac{s}{h^{2}-a^{2}}-\frac{2 h}{h^{2}-a^{2}}
$$


The function of $s$ has a minimum at

$$
s=\left[2\left(h^{2}-a^{2}\right)\right]^{1 / 2} .
$$

The two possible extremal cases occur according to whether relation (11) satisfies $|g|<a$, i.e. $(h+a) \geqq\left[2\left(h^{2}-a^{2}\right)\right]^{1 / 2}$. Bound $(2 b 1)$ is obtained by substitution of $s=h+a$, its maximum permissible value and (2b2) by substitution of value (11).

COROLlary. $V_{\mu}$ is a convex curve if $h \geqq 2^{1 / 2} a$.

This follows from bound (2b2).

THEOREM 2. Let, in addition to the hypothesis of Theorem 1, the assumption be made that the angle $\delta$ between the normal to $V_{\mu}$ and the line joining the point $w$ to the origin be given, then the bounds of $K(w)$ are sharpened as follows:

$(12 \mathrm{~b} 1) \quad(\operatorname{sgn} c) K \geqq\left(h \cos \delta-\left(a^{2}-h^{2} \sin ^{2} \delta\right)^{1 / 2}\right) /\left(h^{2}-a^{2}\right)$ for $h \geqq 3 a /\left(1+8 \sin ^{2} \delta\right)^{1 / 2}$,

$(12 \mathrm{~b} 2) \quad(\operatorname{sgn} c) K \geqq\left(-h \cos \delta+\left(2\left(h^{2}-a^{2}\right)^{1 / 2}\right) /\left(h^{2}-a^{2}\right) \quad\right.$ for $h \leqq 3 a /\left(1+8 \sin ^{2} \delta\right)^{1 / 2}$.

Corollary. $V_{\mu}$ is convex if $h \geqq 2^{1 / 2} a /\left(1+\sin ^{2} \delta\right)^{1 / 2}$.

Proof. The method of proof follows along the lines of the proof of Theorem 1 . The term $h \cos \delta$ must be substituted in (9) and (10) in place of $h$ and the allowed values of $s$ are given by

$$
h \cos \delta-\left(a^{2}-h^{2} \sin \delta\right)^{1 / 2} \leqq s \leqq h \cos \delta+\left(a^{2}-h^{2} \sin ^{2} \delta\right)^{1 / 2} .
$$

3. Determination of functions with given representations. In this section it will be shown that lemniscates, certain rational functions and the level curves of Green's function can be represented so as to satisfy the hypotheses of Theorems 1 and 2.

LEMMA 1. Let

$$
\begin{array}{rlrl}
P(w) & =\prod_{i=1}^{n}\left(w-\alpha_{i}\right)^{m_{i}}, & & m_{i}>0, \\
\left|\alpha_{i}\right| & \leqq a, & i=1,2, \ldots, n .
\end{array}
$$

Then the representation (1) holds with $c>0$.

This lemma was proved by Walsh [9], [3]. It follows that the curvature of the lemniscate $V_{\mu}:|P(w)|=\mu$ satisfies the estimates of Theorems 1 and 2 with $c>0$.

LEMMA 2. Let

$$
R(w)=\prod_{i=1}^{n} 1 /\left(w-\beta_{i}\right) \quad \text { with }\left|\beta_{i}\right|<a ;
$$

then $R^{\prime} / R$ has the required representation (1) with $c<0$. 
The proof of the lemma depends only on the logarithmic derivative and the result follows from Lemma 1 . The curvature of the level curve of $R(w)$ satisfies Theorems 1 and 2 with $\operatorname{sgn} c<0$. This result can also be obtained as a special case of the curvature of the level curve of general rational functions derived by the author [5].

Lemma 3. Let $P(w)$ have the representation (1) with $c=1$. Then $V_{\mu}$ is the level curve of the Green's function of the region $|P(w)|>\mu$, with pole at infinity.

Proof. Let $F(w)=\log P(w)-\log \mu, F(w)$ is analytic in $|w|>a$. By hypothesis $F^{\prime}(w)=P^{\prime} \mid P=1 /(w-g(w))$ exists and is analytic in $|w|>a, \operatorname{Re} F(w) \rightarrow \log |w|$ as $w \rightarrow \infty, \operatorname{Re} F(w)=0$ for $|P(w)|=\mu$; therefore $G(u, v)=\operatorname{Re} F(w)$ is the Green's function of $|P(w)|>\mu$ with pole at $\infty$.

LEMMA 4. Let $G(u, v)$ be the Green's function with pole at $\infty$ for a region $R$ whose boundary is contained in $|w| \leqq a$. Let $H$ be the conjugate function, $\phi=G+i H$, then $\phi^{\prime}(w)$ has the representation (1).

Proof. If $B$ is a Jordan configuration, the Green's function has the following representation [8, pp. 270-272]:

$$
G(z)=G\left(x_{0}, y_{0}\right)=\int_{B} \log |(z-\zeta)| d \sigma+g ;
$$

$g$ is Robin's constant, $d \sigma>0, \int_{B} d \sigma=1, \zeta$ is a point on $B, z \in R$.

If $B$ is not a Jordan configuration integrate instead over a level curve $G_{\mu}, \mu \rightarrow 0$. Similarly,

$$
\begin{aligned}
H(z) & =\int_{B} \arg (z-\zeta) d \sigma \\
\phi(z) & =\int_{B} \log (z-\zeta) d \sigma+g \\
\phi^{\prime}(z) & =\int_{B} \frac{d \sigma}{z-\zeta}, \quad \phi^{\prime}(z) \text { analytic for }|z|>a .
\end{aligned}
$$

Following the argument used in [3] for the proof of Lemma 1 we have, for fixed $z$, $|z|>r$, the function $1 /(1-w / z)$ which maps $|w| \leqq r$ into the interior of a circle which does not include the origin. Therefore there exists a unique value $g(z),|g(z)| \leqq a$, such that

$$
\phi^{\prime}(z)=\frac{1}{z} \int \frac{1}{1-\zeta / z} d \sigma=\frac{1}{z(1-g(z) / z)} .
$$

The methods of this paper can also be extended to the level curves of general rational functions and harmonic functions. We will consider the curvature of level curves $U_{\mu}:\{w|| P(w) \mid=\mu\}$ where the logarithmic derivative of $P(w)$ has the representation

$$
\frac{P^{\prime}(w)}{P(w)}=c\left(\frac{1}{w-g_{1}(w)}-\frac{1}{w-g_{2}(w)}\right)
$$


where $c>0, \zeta_{i}$ arbitrary complex constants $a_{i}>0$, for $i=1,2$;

$$
g_{i}(w) \in D_{i}:\left\{w|| w-\zeta_{i} \mid \leqq a_{i}\right\}
$$

and the $g_{i}(w)$ are analytic exterior to $D_{1} \cup D_{2}$.

A rational function

$$
R(w)=\prod_{i=1}^{n}\left(w-\alpha_{i}\right) / \prod_{i=1}^{n}\left(w-\beta_{i}\right),
$$

where all the zeros $\alpha_{i}$ lie in the disc $D_{1}$ and the poles $\beta_{i}$ lie in the disc $D_{2}$, has the required representation. This follows from coincidence Lemma 1.

The representation can also be extended to functions which are harmonic in a region and assume the values 0 and 1 respectively on a set of curves contained in disjoint discs $D_{1}$ and $D_{2}$.

Calculations of the curvature can be carried out using the methods of proof of Theorem 1 and the estimates derived previously by the author [5] are obtained by this shorter method.

4. The curvature of the level curves of lacunary polynomials. This section deals with the curvature of level curves of polynomials which satisfy additional symmetry conditions on the location of its zeros. For a polynomial with $p$ missing coefficients the following estimates are obtained:

THEOREM 3. Let the zeros $\alpha_{i}$ of the polynomial $P(w)=\prod_{i=1}^{n}\left(w-\alpha_{i}\right),\left|\alpha_{i}\right| \leqq a$, satisfy the additional condition $\sum_{k=1}^{n} \alpha_{k}^{l}=0$ for $l=1,2, \ldots, p$; then the curvature $K$ of $V_{\mu}$ satisfies the following sharp inequalities for $|w|=h>a$ :

$$
\begin{gathered}
K \geqq \frac{h^{p+1}-p a^{p+1}}{h^{p}\left(h^{p+1}+a^{p+1}\right)} \text { for } 2 a\left(h^{p+1}+a^{p+1}\right) \leqq(1+p)\left(h^{2}-a^{2}\right) h^{p}, \\
K \geqq 2 h^{p}\left[h^{2 p+2}-a^{2 p+2}+\left(h^{2}-a^{2}\right)(1+p) h^{p} a^{p}\right]^{1 / 2} \\
-2 h^{2 p+1}-p\left(h^{2}-a^{2}\right) h^{p-1} a^{p} \quad \text { for } 2 a\left(h^{p+1}+a^{p+1}\right) \geqq(1+p)\left(h^{2}-a^{2}\right) h^{p}, \\
K \leqq \frac{h^{p+1}+p a^{p+1}}{h^{p}\left(h^{p+1}-a^{p+1}\right)} .
\end{gathered}
$$

Proof. An improved form of the coincidence lemma due to Rubinstein and Walsh [3] is applied in the proof. It states that for a polynomial with the hypothesis of the theorem we have the representation

$$
\frac{P^{\prime}(w)}{P(w)}=\frac{c}{w-g(w)} \text { with }|g(w)| \leqq \frac{a^{p+1}}{h^{p}} \text { for }|w|=h>a, c>0 .
$$

The inequalities (5) derived in the proof of Theorem 1 again form the starting point of the calculations of curvature.

The basis of the proof is the reformulation of the coincidence lemma (14) so that the inequality (6) for the absolute value of the derivative of a bounded analytic function can again be applied. 
We rewrite (14) in the form $\left|(w / a)^{p} g(w)\right| \leqq a$, for $h>a$ and obtain

$$
\left|p g(w) / w+g^{\prime}(w)\right| \leqq\left(a^{p+2}-|g(w)|^{2} h^{2 p} / a^{p}\right) / h^{p}\left(h^{2}-a^{2}\right) .
$$

From (4) we derive the bounds

$$
\left(1-\left|p g(w) / w+g^{\prime}(w)\right|+\operatorname{Re} p g(w) / w\right) / s \leqq K \leqq\left(1+\left|p g(w) / w+g^{\prime}(w)\right|+|p g(w) / w|\right) / s,
$$

$\operatorname{Re} p g / w \geqq p(h-s) / h$. Substituting (15) we obtain

$$
\left(h^{2}-a^{2}\right) h^{p} a^{p} s K \geqq h^{2 p}|g(w)|^{2}-a^{2 p+2}+\left(h^{2}-a^{2}\right)(1+p) h^{p} a^{p}-p s\left(h^{2}-a^{2}\right) h^{p-1} a^{p} .
$$

These bounds are again reduced to a function of $s$ alone by means of the identity (8). With calculations similar to those employed in the proof of Theorem 1 we obtain for the lower bound,

$$
\begin{aligned}
& K\left(h^{2}-a^{2}\right) h^{p} a^{p} \\
& \geqq\left[h^{2 p+2}-a^{2 p+2}+\left(h^{2}-a^{2}\right)(1+p) h^{p} a^{p}\right] / s+h^{2 p} s-2 h^{2 p+1}-p\left(h^{2}-a^{2}\right) h^{p-1} a^{p}, \\
& d K / d s=0 \text { for } s_{0}=\left[h^{2 p+2}-a^{2 p+2}+\left(h^{2}-a^{2}\right)(1+p) h^{p} a^{p}\right]^{1 / 2} / h^{p} .
\end{aligned}
$$

By the coincidence lemma, the minimum value of $K$ lies at turning point (bound (13a2)) if

$$
s_{0} \leqq h+a^{p+1} / h^{p}, \quad \text { i.e. } 2 a\left(h^{p+1}+a^{p+1}\right) \geqq\left(h^{2}-a^{2}\right)(1+p) h^{p} .
$$

The bound (13a1) is obtained by substituting the largest permissible bound for $s$; $s=h+a^{p+1} / h^{p}$.

Inequality (16) is satisfied for values of $h$ close to $a,(h / a<\sqrt{ } 2)$, bound (13a1) holds for large values of $h / a$.

The upper bound can be obtained by similar methods, the function of $s$ is monotonically decreasing and the smallest permissible value of $s$ substituted. It is also obtained by application of a lemma due to Rubinstein [3].

For a point on the positive real axis, i.e. $w=h$, the lemniscate $\left|w^{p+1}-a^{p+1}\right|=\mu$ has the curvature (13b) and the lemniscate $\left|w^{p+1}+a^{p+1}\right|=\mu$ assumes the minimum curvature (13a1).

If the point $w$ approaches the boundary, i.e. $h \rightarrow a$, the bound (13b) approaches $1 /(h-a)$ and the bound (13a2) approaches $-1 /(h-a)$ which coincides with the estimate due to Walsh [7] for the $\alpha_{k}$ restricted to lie in a half-plane instead of a disc.

For the case $p=1$, i.e. the center of gravity of the zeros is prescribed, the bound (13a2) can be simplified as follows:

$$
h\left(h^{2}-a^{2}\right) K \geqq 2 a\left[h^{2}-a^{2}\right]^{1 / 2}(h+a)-2 h^{3}-a\left(h^{2}-a^{2}\right) \quad \text { for } a\left(h^{2}+a^{2}\right) \geqq h\left(h^{2}-a^{2}\right) .
$$

COROllary. $V_{\mu}$ is convex if

$$
2 h^{p}\left[h^{2 p+2}-a^{2 p+2}+\left(h^{2}-a^{2}\right)(1+p) h^{p} a^{p}\right]^{1 / 2} \geqq 2 h^{2 p+1}+p\left(h^{2}-a^{2}\right) h^{p-1} a^{p} .
$$

This follows by requiring the bound (13a2) to be nonnegative. For $p=1$, this reduces to the condition $4 h^{2}\left(h^{2}-a^{2}\right)-a\left(h^{2}+a^{2}\right) \geqq 0$ with solution for $h / a<\sqrt{ } 2$, 
which is sharper than that obtained without prescribing the center of gravity. As $p$ increases the lemniscate $V_{\mu}$ will be convex for decreasing values of $h$.

Estimates of the curvature of the orthogonal trajectories of lacunary polynomials can be obtained by similar methods. We have

THEOREM 4. With the hypotheses of Theorem 3 the curvature $K$ of the level curves $\arg P(w)=$ const satisfies the inequality

$$
|K| \leqq \frac{-2 h^{p}\left[h^{2 p+2}-a^{2 p+2}\right]^{1 / 2}+2 h^{2 p+1}+p\left(h^{2}-a^{2}\right) a^{p} h^{p-1}}{a^{p} h^{p}\left(h^{2}-a^{2}\right)} .
$$

Proof. The starting point is the formula for the curvature of images of radial straight lines

$$
K(w)=\left|P^{\prime}(w) / P(w)\right| \operatorname{Im}\left\{-P P^{\prime \prime} \mid P^{\prime 2}\right\} .
$$

For curves with given representation (1),

$$
(\operatorname{sgn} c) K(w)=\operatorname{Im}\left\{g^{\prime}(w)\right\} /|w-g(w)| .
$$

From this we obtain the bounds

$$
|K(w)| \leqq \frac{|p g / w+g|+p \operatorname{Im}\{(w-g) / w\}}{|w||(w-g) / w|} .
$$

The same coincidence lemma, identity (8), and similar simplifications lead to

$$
a^{p} h^{p}\left(h^{2}-a^{2}\right)|K(w)| \leqq-\left(h^{2 p+2}-a^{2 p+2}\right) / s-h^{2 p} s+2 h^{2 p+1}+p\left(h^{2}-a^{2}\right) a^{p} h^{p-1} .
$$

Substitution of the extremal values for $s, s=\left[h^{2 p+2}-a^{2 p+2}\right]^{1 / 2} / h^{p}$, yields the bound (17).

5. Application to the lemniscate $L_{1}$. The previous sections dealt with properties of level curves in a single plane; in this section these results are connected with the radius of the lemniscate. Calculations are carried out for the lemniscate

$$
L_{1}:|P(w)|=\prod_{k=1}^{n}\left|w-\alpha_{i}\right|=1 .
$$

The results can be scaled up for arbitrary constants. An upper bound is derived for the radius of the disc containing the zeros which implies convexity of $L_{1}$.

THEOREM 5. Let the zeros of $L_{1}$ be inscribed in a disc with radius a and center at the center of gravity of the zeros; then $L_{1}$ is convex if a satisfies the following inequality:

$$
4\left(1-a^{2}\right)^{3 / 2}\left(1-2 a^{2}\right) \geqq a .
$$

Proof. $w_{0}=(1 / n) \sum_{k=1}^{n} \alpha_{k}=0$ by hypothesis. By a lemma due to Pommerenke [2, p. 99] this implies $h^{2} \geqq 1-a^{2}$. The convexity condition for $p=1$ is 
$4 h^{2}\left(h^{2}-a^{2}\right) \geqq a^{3}-h^{2} a$. Inequality (19) is obtained by combining these two inequalities. The solution to (19) lies between $3^{-1 / 2}$ and $2^{-1 / 2}$, and thus represents a slight improvement over the author's previous result which implies the bound $a \leqq 3^{-1 / 2}$ for $w_{0}=0[6]$.

\title{
BIBLIOGRAPHY
}

1. Z. Nehari, Conformal mapping, McGraw-Hill, New York, 1952. MR 13, 640.

2. Ch. Pommerenke, On metric properties of complex polynomials, Michigan Math. J. 8 (1961), 97-115. MR 27 \#1564.

3. Z. Rubinstein and J. L. Walsh, Extension and some applications of the coincidence theorems, Trans. Amer. Math. Soc. 146 (1969), 413-427. MR 40 \#4428.

4. Dorothy B. Shaffer, Distortion theorems for lemniscates and level loci of Green's functions, J. Analyse Math. 17 (1966), 59-70. MR 36 \#361.

5. - Distortion theorems for the level curves of rational functions and harmonic functions, J. Math. Mech. 19 (1969), 41-48.

6. - On the convexity of lemniscates, Proc. Amer. Math. Soc. 26 (1970), 619-620.

7. J. L. Walsh, Lemniscates and equipotential curves of Green's function, Amer. Math. Monthly 42 (1935), 1-17.

8. - The location of critical points of analytic and harmonic functions, Amer. Math. Soc. Colloq. Publ., vol. 34, Amer. Math. Soc., Providence, R. I., 1950. MR 12, 249.

9. - A theorem of Grace on the zeros of polynomials revisited, Proc. Amer. Math. Soc. 15 (1964), 354-360. MR 28 \#4092.

\author{
FAIRFIELD UNIVERSITY, \\ Fairfield, Connecticut 06430
}

